\title{
INTERPRETIVE SUMMARIES, NOVEMBER 2012
}

Invited review: Anaerobic fermentation of dairy food wastewater. By Hassan and Nelson, page 6188. From environmental and financial perspectives, dairy food waste remains an area of concern. Fermentor designs that maintain a high loading of organisms are the most successful. Surfactant, temperature, carbon:nitrogen ratio, hydraulic retention time, $\mathrm{pH}$, and hydrogen pressure are important factors influencing methane production. The microflora within an anaerobic digester is very complex and not fully identified. As anaerobic digesters are utilized in more dairy plants, additional research should be directed towards selecting the best cultures that maximize methane production from dairy foods waste. Anaerobic digestion is a valid solution for the disposal of dairy foods waste.

Effect of ultraviolet light on water- and fat-soluble vitamins in cow and goat milk. By Guneser and Yuceer, page 6230. Pasteurization is widely used as a thermal process for milk. However, heat treatment may damage some vitamins in milk. In this study, ultraviolet (UV) light as a nonthermal treatment was applied to cow and goat milk samples to determine the effects on some water- and fat-soluble vitamins. Amounts of water and fat-soluble vitamins in milk were affected by pasteurization and UV light treatment. Vitamins C and E were more sensitive to UV light than others. Before recommending UV light treatment as an alternative to heat treatment, more studies on the effectiveness of UV light on nutritional value of milk should be investigated.

Oxidative stability of an extended shelf-life dairy-based beverage system designed to contribute to heart health. By Moore et al., page 6242. Four formulations of an ultra-high-temperature (UHT)pasteurized dairy-based beverage system, fortified with varying levels of fish oil, were produced. Physical, microbiological, and chemical quality parameters were stable over the 35 -d refrigerated storage period. The dairy product, which was not different in aroma compared with a commercially available UHT milk product, delivered $432 \mathrm{mg}$ of n-3 fatty acids per serving after $35 \mathrm{~d}$ of storage.

Effect of xylitol on the functional properties of low-fat process cheese. By Kommineni et al., page 6252. Fat is a critical component of process cheese and plays an important role in its functional characteristics. Consequently, low-fat process cheese typically has functional problems such as a firm, rubbery texture and poor melting characteristics. In the present work, a low-fat process cheese was developed with improved functional properties that utilized xylitol as a fat replacer. Xylitol is a hygroscopic sugar alcohol and improves the texture of low-fat process cheese by providing lubricity to the protein network in process cheese.

Combined effect of heat treatment and ionic strength on the functionality of whey proteins. By Hussain et al., page 6260. Whey proteins are a valuable source of milk proteins containing high nutritional value and varied functional properties such as gelling or emulsifying. The emphasis of this study was to relate the rheological and morphological properties of heator salt-induced gels of whey proteins to the particle size, hydrophobicity, and interactions of the protein molecules in the gel network. From an industrial point of view, our project earns vital significance due to lack of basic data regarding strong interactions between ionic strength and dynamic thermal treatments effecting protein functional properties. A careful adjustment of these fractions may be helpful for dairy products improvement.

Survival of microencapsulated probiotic Lactobacillus paracasei LBC-1e during manufacture of Mozzarella cheese and simulated gastric digestion. By Ortakci et al., page 6274. To provide health benefits, probiotic bacteria need to reach the intestinal tract in sufficient viable numbers. Generally, high numbers of bacteria are killed as they pass through the stomach. It is important to know their survival during food manufacture, storage, and gastric digestion to determine how many probiotic bacteria need to be added to the food. We found that both free bacteria and gel-encapsulated Lactobacillus paracasei bacteria survived the hot stretching process during manufacture of Mozzarella cheese. However, the alginate capsules did not provide any additional protection above that provided by cheese during simulated gastric digestion using hydrochloric acid.

Effect of the high-pressure-release phase on the protein composition of the soluble milk fraction. By Bravo et al., page 6293. High pressure is a novel, nonthermal, and environmentally friendly food processing technology. Pressurized food looks like fresh food, and is highly appreciated by the consumer. Pressurization of milk is an active and growing area of research, and the effect of high pressure on milk proteins has been widely studied, taking into account different factors such as protein concentration, pressure level, treatment time, and so on, whereas other aspects, such as the effect of pressure release rate have not yet been studied in depth. This work shows the effect of the pressure release phase on casein micelle disruption and whey protein denaturation in milk. 
Behavior of Enterobacter pulveris in amorphous and crystalline powder matrices treated with supercritical carbon dioxide. By Callanan et al., page 6300. This paper describes supercritical carbon dioxide $\left(\mathrm{scCO}_{2}\right)$ treatment of different types of food powders inoculated with Enterobacter pulveris, a microbe that is a typical contaminant in dry powder products such as infant formula. The $\mathrm{scCO}_{2}$ treatment did not significantly reduce bacterial numbers in powders composed of amorphous materials. However, the bacterium was found to be much less resistant to $\mathrm{scCO}_{2}$ and heat treatment in powders composed of crystalline material. This information is important in designing strategies to decontaminate food powders.

Bovine milk fat globule membrane affects virulence expression in Escherichia coli O157:H7. By Tellez et al., page 6313. The bovine milk fat globule membrane (MFGM) is a supramolecular structure, composed of phospholipids and protein, which surrounds the fat globules in milk. The components of the MFGM have been associated with several healthpromoting properties such as anticancer, anticholesterolemic, and antimicrobial effects. For the first time, a specific effect of the MFGM is reported against Escherichia coli $\mathrm{O} 157: \mathrm{H} 7$, an important foodborne pathogen. We showed that MFGM downregulates the expression of Shiga toxin genes and other virulence genes. The processing history of the MFGM modifies this effect.

Usefulness of a large field of view sensor for physicochemical, textural, and yield predictions under industrial goat cheese (Murcia al Vino) manufacturing conditions. By Rovira et al., page 6320. A light backscatter sensor with a large field of view (LFV) was used for the on-line monitoring of goat cheese coagulation and syneresis in industrial conditions. The LFV reflectance ratio provided good results for predicting the moisture content, yield and texture of the final cheese. By means of the LFV sensor and applying an equation developed in this study, moisture content could be controlled in industrial conditions with a low standard error, confirming the suitability of this prototype sensor for curd moisture content standardization before ripening. Cheese manufacturing effectiveness and profitability may be improved as a result.

Exopolysaccharides modify functional properties of whey protein concentrate. By Deep et al., page 6332. The objective of this research was to produce a whey protein concentrate (WPC) with modified functionality using exopolysaccharide (EPS)- producing cultures. Whey protein concentrate fermented with cultures producing EPS was added to whey before or after ultrafiltration. Ultrafiltered whey containing EPS was used in making WPC. The presence of EPS in whey improved solubility, gelation, emulsification, and reduced protein denaturation in WPC. The type of EPS was one of the factors affecting WPC functionality. Cooling of the culture containing EPS prior to its addition to whey further improved the functional properties of the resulting WPC.

Heat stability of micellar casein concentrate as affected by temperature and pH. By Sauer and Moraru, page 6339. This work is aimed at generating information about the heating behavior of micellar casein concentrate across a range of $\mathrm{pH}$ and temperature conditions, in order to understand their heat stability during commercial sterilization. The generated knowledge will provide the dairy and food industry with data necessary for effectively stabilizing micellar casein preparations and developing practical applications for this new dairy ingredient, particularly the production of shelf-stable protein beverages.

The trade network in the dairy industry and its implication for the spread of contamination. By Pinior et al., page 6351. The influence of vertical and horizontal trade structures between milk producers, dairies, and consumers on the potential spread of a hypothetical agent was investigated using a computer simulation model. Particular attention was paid to the effect of the magnitude of horizontal flow of goods between dairies on the spread of a contagion. The simulation results indicate that even a small increase of the horizontal trade leads to a substantial increase in the potency of spread of contamination.

The influence of feed and herd on fatty acid composition in 3 dairy breeds (Danish Holstein, Danish Jersey, and Swedish Red). By Poulsen et al., page 6362. The composition of milk fat in dairy cows is related to both genetic and environmental factors. Here, the effect of feed and herd on fatty acid (FA) composition in milk was examined in 3 Scandinavian breeds: Danish Holstein-Friesian, Danish Jersey, and Swedish Red. Distinct FA profiles for all breeds were revealed and related to the pronounced regional differences in feed between countries. Furthermore, the proportion of phenotypic variation that could be explained by herd was calculated and the herd effect for individual FA was generally lower for Danish Holstein compared with the 2 other breeds.

Commensal symbiosis between a Lactococcus lactis strain and an Enterococcus mundtii strain increases cell yield in constituted broth. $B y K i$ moto-Nira et al., page 6372. In developing new starter cultures, we investigated the synergistic effect between Enterococcus mundtii IFO 13712 and Lactococcus lactis strains, many of which are widely used as starter bacteria for making dairy products and have probiotic properties. The growth yield of a mixed culture of $L$. 
lactis strain Y and IFO 13712 in de Man, Rogosa, and Sharpe broth was greater than that of either single culture. The effect was commensalism for strain Y, and Tween 80 and citrate in the broth contributed to the commensalism.

Short communication: Simultaneous analysis of reducing sugars and 5-hydroxymethyl-2-furaldehyde at a low concentration by high performance anion exchange chromatography with electrochemical detector, compared with HPLC with refractive index detector. By $Y u$ et al., page 6379. High performance anion exchange chromatography with electrochemical detector using an anion exchange column could simultaneously test reducing sugars and 5-hydroxymethyl-2-furaldehyde, especially for a trace analysis. The lowest detectable limit of reducing sugars and 5-hydroxymethyl-2-furaldehyde was $0.00005 \mathrm{~mol} / \mathrm{L}$. High performance liquid chromatography with refractive index detector using a cation exchange column was not suitable to test for 5-hydroxymethyl-2-furaldehyde, which always produces a tailing peak.

Hot topic: Association of telomere length with age, herd, and culling in lactating Holsteins. By Brown et al., page 6384. Telomeres provide a buffer on the end of chromosomes that allow for complete replication of DNA and protect chromosomal integrity. Telomeres are reported to erode prematurely under stress in humans. Variation in telomere length was demonstrated among 201 Holstein cows from 10 herds. Telomere length was shorter for older cows and varied significantly among herds. Short telomeres were associated with elevated culling levels. Telomere length evaluation has potential to provide a stable and quantitative measure of stress levels indicative of a cow's physiological response to stress.

Preference for pasture versus freestall housing by dairy cattle when stall availability indoors is reduced. By Falk et al., page 6409. Access to pasture is typically considered positive for cattle welfare. This study evaluated the preference for pasture by varying the number of lying places available inside the barn while providing free access to pasture. Cows showed a partial preference for pasture versus freestall housing; pasture use was influenced by the time of day and environmental conditions such as temperature and rainfall. Reduced freestall availability did not increase time spent outside, but did reduce lying time inside the barn.

Relationship between daily rumination time and estrus of dairy cows. By Reith and Hoy, page 6416. Measurement of rumination is usually used for monitoring of diet composition adequacy and for early detection of metabolic disorders. The aim of the study was to verify a relationship between rumination time and estrus. Rumination time was significantly reduced at the day of estrus. Thus, reduced rumination indicates metabolic disorders and may also be an indication of estrus.

Factors determining milk quality and implications for production structure under somatic cell count standard modification. By Dong et al., page 6421. We modeled determinants of somatic cell count (SCC) in the United States and conducted empirical analysis based on national farm-level data from 2005 . Location outside the southeastern United States, lower herd age, full-time farming status, use of biosecurity guidelines, good milking facilities and operations management, and application of related quality tests were all associated with lower SCC levels. Herds that did not meet the 400,000 cells/mL standard were generally smaller, more likely to be family owned, had less unit revenue per cow, and were more likely to exit farming in $10 \mathrm{yr}$ than herds meeting the standard.

Bayesian estimation of the diagnostic accuracy of a multiplex real-time PCR assay and bacteriological culture for 4 common bovine intramammary pathogens. By Paradis et al., page 6436. Intramammary infections are traditionally diagnosed by bacteriological culturing, which lacks sensitivity. Polymerase chain reaction (PCR) is a faster diagnostic technique and is potentially more sensitive. Our objective was to compare false-positive and false-negative likelihood between these 2 tests in detecting, in milk, bacteria that cause intramammary infection. Both techniques were comparable. However, mastitis severity influenced the performance of both methods for detection of Staphylococcus aureus and Escherichia coli. For clinical samples, PCR sensitivity decreased by more than $10 \%$. These results improve our knowledge about the relative performance of these tests, allowing us to integrate the decision-making process at the farm to reduce the likelihood of erroneous results.

Validation of handheld meters to measure blood L-lactate concentration in dairy cows and calves. By Burfeind and Heuwieser, page 6449. Handheld devices are becoming more common in veterinary diagnostics. The objective was to validate a handheld meter for an immediate determination of lactate concentration in dairy cows and calves and to study the effect of different anticoagulants on test results. Lactate was measured in various conditions in cattle. Blood lactate concentration was measured reliably with the handheld meter in dairy cows and calves. However, the anticoagulant used can influence the results considerably.

Infection dynamics of digital dermatitis in firstlactation Holstein cows in an infected herd. By 
Capion et al., page 645\%. Digital dermatitis continues to be an important cause of lameness and production losses in dairy herds. In this study, the spread of digital dermatitis was monitored in an endemically infected commercial dairy herd, specifically following 39 healthy heifers as they were introduced to the group of lactating cows. Three categories of susceptibility to digital dermatitis in cows were identified. The most interesting category consisted of $28 \%$ consistently healthy cows. Advancing age at calving increased the odds of susceptibility to digital dermatitis. There was no relation between time since infection, time since calving, and Treponema spp. phylotypes present in digital lesions.

Accuracy of in-line milk composition analysis with diffuse reflectance near-infrared spectroscopy. By Melfsen et al., page 6465. The knowledge of daily milk composition of individual cows can be helpful for improved cow or herd management. Near-infrared spectroscopy (NIRS) has the potential to analyze milk samples in-line during the milking process. In this study, the accuracy of a special designed in-line milk analysis with diffuse reflectance NIRS was investigated. Good and excellent results were achieved with regard to calibration and validation of 5 tested milk constituents: fat $(\%)$, protein $(\%)$, lactose $(\%)$, urea $(\mathrm{mg} / \mathrm{L})$, and somatic cell count. The standard error of prediction of the predicted milk constituents was compared with accuracy recommendations for in-line milk-analyzing tools. Both evaluation procedures confirmed that the in-line analyzing device tested was suitable for predicting those milk constituents with adequate accuracy.

Environmental contamination with Mycobacterium avium subspecies paratuberculosis within and around a dairy barn under experimental conditions. By Eisenberg et al., page 6477. Johne's or Mycobacterium avium ssp. paratuberculosis (MAP) control programs advise separation between dairy cows and susceptible calves. This study investigated the levels of MAP present at different locations in and around a dairy barn purposely stocked with MAP-shedding cattle. Data showed similar MAP presence inside the barn in feces-contaminated areas as in areas contaminated only with dust. Outside the barn, MAP was present significantly less frequently. Preventing MAP exposure of youngstock may require housing them in separate buildings. Therefore, current Johne's control programs could include housing youngstock in separate barns as an additional management measure.

Examining the impact of intramammary infections with minor mastitis pathogens on the acquisition of new intramammary infections with major mastitis pathogens-A systematic review and meta-analysis. By Reyher et al., page 6483.
Major mastitis pathogens are usually considered more virulent and damaging than minor mastitis pathogens. A number of studies exist with conflicting results as to whether intramammary infections with minor pathogens reduce, increase, or have no effect on the risk of a quarter acquiring a new intramammary infection with a major pathogen. We conducted a systematic review and meta-analysis of the available literature and found significant variability among studies. Overall, challenge studies and smaller studies showed protective effects, whereas observational studies showed no effect. Variables explaining the heterogeneity among studies and accounting for the differences in effects reported were investigated.

The effect of incomplete milking or nursing on milk production, blood metabolites, and immune functions of dairy cows. By Carbonneau et al., page 6503. Reducing the amount of milk harvested during the first $5 \mathrm{~d}$ of lactation either by milking the cows incompletely twice a day or by leaving the cows with their calves and milking them once daily on d 3, 4, and 5 reduces metabolic stress without compromising the productivity of high-yielding dairy cows.

Pregnancy per artificial insemination after presynchronizing estrous cycles with the Presynch-10 protocol or prostaglandin $F_{2 \alpha}$ injection followed by gonadotropin-releasing hormone before Ovsynch-56 in 4 dairy herds of lactating dairy cows. By Stevenson and Pulley, page 6513. Presynchronization of estrous cycles by administering prostaglandin $\mathrm{F}_{2 \alpha}(\mathrm{PG}) 3 \mathrm{~d}$ before gonadotropin-releasing hormone (PG-3-G) compared with 2 injections of $P G$ $14 \mathrm{~d}$ apart (Presynch-10) tended to increase pregnancy per $\mathrm{AI}(\mathrm{P} / \mathrm{AI})$ during all seasons of the year in 4 dairy herds in which a timed AI protocol was initiated $10 \mathrm{~d}$ after the second (Presynch-10) or only presynchronization PG (PG-3-G) injection. Cows inseminated early at estrus during cooler parts of the year before the scheduled timed AI had reduced P/AI compared with those on the PG-3-G treatment. The PG-3-G treatment, however, produced more P/AI than Presynch-10 during summer, but not during cooler seasons.

Validation of an automated method to count steps while cows stand on a weighing platform and its application as a measure to detect lameness. By Chapinal and Tucker, page 6523. The objectives were to validate an automated method to count steps from weight distribution measurements and to assess the accuracy of frequency of steps in detecting lameness. The threshold of weight applied to a leg to define a step was determined and used to develop an algorithm to count steps. The frequency of steps taken with the rear legs predicted lameness and correlated 
with other measures of weight shifting among legs. Stepping behavior can be measured automatically and shows promise as a tool to assess lameness.

Short communication: Assessing urea transport from milk to blood in dairy cows. By Spek et al., page 6536. The milk urea concentration (MUC) is used in practice to predict urinary nitrogen excretion of dairy cattle. More insight into daily variation in MUC, partly due to transport of urea between blood and milk, may increase the usefulness of MUC as predictor of urinary nitrogen excretion. This study aimed to provide insight in urea transport characteristics from milk to blood. The current study showed that urea is transported from milk to blood and indicates that the rate of transport may be related to MUC.

Short communication: Performance of a blocking antibody ELISA bulk-tank milk test for detection of dairy sheep flocks exposed to border disease virus. By Corbière et al., page 6542. Test characteristics of a blocking antibody ELISA applied to bulk-tank milk samples were investigated in 161 dairy sheep flocks free from or exposed to border disease virus. The best decision threshold was different from the one currently used in dairy cattle. Test sensitivity and specificity were sufficiently high $(>90 \%)$ to allow for its use as a powerful tool in Pestivirus control schemes in dairy sheep flocks.

Short communication: Lying behavior of lactating dairy cows is influenced by lameness especially around feeding time. By Yunta et al., page 6546. Lameness is a common economic and welfare problem in dairy herds. Early detection of lame animals can be facilitated through automatic measures of changes in behavior. The objective of the study was to compare lying behavior of sound and moderately lame cows and to find some alternatives to detect lameness. Paying attention to those cows that remain lying down around the time when fresh feed is delivered seems an effective way to detect moderately lame cows.

Technical note: Validating a dynamometer for noninvasive measuring of udder firmness in dairy cows. By Bertulat et al., page 6550. High-producing dairy cows face high intramammary pressure and udder firmness. A clinical examination by palpating the udder, however, provides only a subjective estimate of pressure and firmness. In the past, several approches have been used to measure udder pressure, but the methods applied were invasive and cumbersome. In this study, we validated an electronical dynamometer for a noninvasive measurement of udder firmness. Following a strict standardized operating protocol, the dynamometer achieves high inter-investigator repeatability and provides an adequate measurement of udder firmness.
Effects of dietary betaine on milk yield and milk composition of mid-lactation Holstein dairy cows. By Peterson et al., page 655\%. Betaine is a feed additive that has been shown to increase milk production and milk fat percentage in lactating dairy cows and goats. The objective of this research was to determine the effects of betaine supplementation on milk yield and milk composition in mid-lactation Holstein dairy cows. Dietary betaine at the highest inclusion rate $(100 \mathrm{~g} / \mathrm{d})$ increased milk yield, whereas milk protein percentage decreased with betaine supplementation. Overall, no change was detected in yields of milk components by inclusion of dietary betaine.

Free ferulic acid uptake in lactating cows. By Soberon et al., page 6563. Free ferulic acid is a phenolic compound that naturally occurs in forages and has antioxidant and anticancer activities. The objective of this study was to investigate the transfer of a given dosage of ferulic acid into the milk of dairy cattle. It was observed that orally ingested ferulic acid can be transferred into milk and is present at basal levels of 4.1 $\mu \mathrm{g} / \mathrm{L}$ in cows fed maize silage. Future research will be required to evaluate the complete health ramifications and sensory and microbiological effects of increased ferulic acid and ferulic acid degradation products in milk.

The effects of social contact and milk allowance on responses to handling, play, and social behavior in young dairy calves. By Duve et al., page 6571. Dairy calves are typically separated from the dam at birth, housed individually, and fed a low milk allowance. This study tested the combined and separate effects of contact with a peer and a high milk allowance, and compared this with staying with the dam. Social contact with the dam reduced responsiveness to restraint, and social contact with a peer increased play and competitive success. High milk allowance increased play in individually housed calves.

The effects of supplementing grazing dairy cows with a partial mixed ration on enteric methane emissions and milk production during mid to late lactation. By O'Neill et al., page 6582. The objective of the current study was to compare enteric methane $\left(\mathrm{CH}_{4}\right)$ emissions and milk production of cows offered a low grass allowance plus partial mixed ration (PMR), a high grass allowance, or a low grass allowance. Enteric $\mathrm{CH}_{4}$ emissions, dry matter intake, and milk production per cow were greatest on the PMR treatment. Treatment had no effect on intensity of enteric $\mathrm{CH}_{4}$ emissions. The greater enteric $\mathrm{CH}_{4}$ emissions per cow on the PMR treatment were due to the increase in dry matter intake rather than to any nutritional characteristic of the PMR. These data provide dairy farmers with information to assist them in determining supplementation strategies to reduce intensity of enteric $\mathrm{CH}_{4}$ emissions. 
Effects of Saccharomyces cerevisiae fermentation product on ruminal starch digestion are dependent upon dry matter intake for lactating cows. By Allen and Ying, page 6591. Effects of Saccharomyces cerevisiae fermentation product (SCFP) on ruminal digestion were determined using ruminally and duodenally cannulated cows in a crossover design experiment with a preliminary period. The SCFP treatment interacted with dry matter intake of cows measured in the preliminary period to affect ruminal digestion rate of starch. Rate of starch digestion decreased with increasing dry matter intake for the SCFP treatment but increased with increasing dry matter intake for the control treatment. Effects of SCFP on ruminal fermentation vary with nutrient demand of cows.

A metabolomics approach to uncover the effects of grain diets on rumen health in dairy cows. By Saleem et al., page 6606. In this study we used quantitative metabolomics to evaluate the effects that feeding graded amounts of barley grain has on the profiles of rumen fluid metabolites in dairy cows. Our data indicate that feeding increasing amounts of barley grain from $0,15,30$, to $45 \%$ was associated with major changes in the profiles of rumen fluid metabolites, suggesting that some of the metabolites identified might be involved in the etiopathology of diseases related to grain-rich diets in dairy cows

Differentiation of mixtures of blend co-product with barley grain based on Fourier transform infrared attenuated total reflection molecular spectroscopy: Carbohydrate molecular spectral profiles and nutritive characteristics in dairy cattle. By Zhang and Yu, page 6624. Effects of blend distillers dried grains with solubles (DDGS) on the molecular structure spectral profile and nutritive characteristics of the carbohydrate in hulless barley. This study aimed to reveal the effects of the inclusion rate of blend DDGS on molecular structure spectral profiles and nutritive characteristics of the carbohydrate in hulless barley in dairy cattle.

Effects of natural and simulated rainfall on indicators of ensilability and nutritive value for wilting alfalfa forages sampled before preservation as silage. By Coblentz and Muck, page 6635. The frustrations associated with conserving high-quality alfalfa silage during periods of unstable or inclement weather are widely known. Within these experiments, rainfall events were applied to wilting alfalfa forages by both simulated and natural methods across 4 independent forage harvests. Based on these experiments, the potential for good fermentation is affected only minimally by single rainfall events applied to relatively wet forages, provided that these events are followed by rapid dehydration to moisture concentrations suitable for making silage. However, attaining acceptable silage fermentations with forages subjected to prolonged exposure under poor drying conditions is likely to be far more problematic.

Low-protein solid feed improves utilization of milk replacer for protein gain in veal calves. $B y$ Berends et al., page 6654. An experiment with 48 veal calves was conducted to quantify the contribution of low protein solid feed to protein and energy retention at 2 stages of the fattening period. Solid feed intake increased the gross efficiency of nitrogen retention in veal calves. This effect was more pronounced at $164 \mathrm{~kg}$ of body weight $(+9.9 \% / \mathrm{g}$ of dry matter solid feed per day) than at $108 \mathrm{~kg}$ of body weight $(+5.4 \% / \mathrm{g}$ of dry matter solid feed per day). Methane production from solid feed when fed in addition to milk replacer was similar to that in ruminants fed only solid feed.

Brown midrib corn silage fed during the peripartal period increased intake and resulted in a persistent increase in milk solids yield of Holstein cows. By Stone et al., page 6665. Conventional (CCS) or brown midrib (BMRCS) corn silage was fed to 70 Holstein cows 3 wk before and 3 wk after parturition, after which cows were switched to a common diet containing only CCS. Production data were collected through wk 15 of lactation. The BMRCS typically contains less lignin and the fiber is more digestible than that in CCS. Cows fed the BMRCS produced more milk and milk solids during the postpartum period, and then continued to produce more protein, lactose, and total solids during the subsequent 12 wk compared with cows fed CCS. Improvements in transition cow performance can extend well into lactation.

An alkalinizing oral rehydration solution containing lecithin-coated citrus fiber is superior to a nonalkalinizing solution in treating 360 calves with naturally acquired diarrhea. By Goodell et al., page 667\%. Dehydration in calves with diarrhea is often treated by administration of an oral rehydration therapy (ORT) solution. The purpose of this study was to compare the efficacy and cost of 2 commercially available ORT solutions in treating dairy calves with naturally acquired diarrhea. Calves that developed diarrhea were assigned randomly to treatment with one of two commercial ORT solutions (Diaque, $\mathrm{n}=180$; Boehringer Ingelheim, Ingelheim, Germany; RE-SORB, $\mathrm{n}=180$; Pfizer Animal Health, New York, NY). Feeding an ORT solution (Diaque) in the milk of calves with diarrhea resulted in a faster resolution of diarrhea, a greater increase in body weight by d 5 of treatment, and decreased treatment costs compared with feeding a different ORT solution (RE-SORB) in conjunction with decreasing milk intake. Our findings support the concept that milk should continue to be fed to calves 
showing mild to moderate signs of diarrhea and dehydration in order to maintain growth.

Effect of exogenous protease enzymes on the fermentation and nutritive value of corn silage. By Young et al., page 668\%. With time in the silo, in vitro ruminal starch digestion of corn silage improves because of degradation of the prolamin matrix from proteolytic mechanisms. This study shows that adding exogenous proteases to chopped corn plants at ensiling has the potential to increase starch digestion in a short period of time without having to wait for the process to occur naturally.

Metabolic characteristics of proteins and biomolecular spectroscopic profiles in different batches of feedstock (wheat) and their co-products (wheat distillers dried grains with solubles) from the same bioethanol processing plant. By Gamage et al., page 6695. Metabolic characteristics of proteins and biomolecular spectroscopic profiles in different batches of feedstock (wheat) and their coproducts (wheat distillers dried grains with solubles, wDDGS) from the same bioethanol processing plant. This study aimed to (1) reveal the metabolic characteristics of proteins in different batches of feedstock of wheat and their co-products (wDDGS) from the same bioethanol processing plant, and (2) characterize the biomolecular spectral profile associated with degradation and digestion in the rumen and intestine in dairy cattle.

Individual animal variability in ruminal bacterial communities and ruminal acidosis in primiparous Holstein cows during the periparturient period. By Mohammed et al., page 6716. Variability in the severity of ruminal acidosis (RA) and in ruminal bacterial community composition (BCC) was investigated in transition cows pre- and postpartum. There were interactions between severity of RA and period, with increased RA severity postpartum for some cows, indicating variability in susceptibility to RA among cows. Ruminal BCC differed between periods for some cows; however, cows demonstrating a greater shift in $\mathrm{BCC}$ were not necessarily those that were most acidotic and vice versa. The study indicated that shifts in BCC were not related to differences in severity of RA.

The effects of viral vaccination of dairy heifer calves on the incidence of respiratory disease, mortality, and growth. By Windeyer et al., page 6731. Although common practice, minimal field research has evaluated preweaning vaccination in dairy heifers. The effect of vaccination of dairy calves on the incidence of bovine respiratory disease and mortality, and on growth was assessed in a randomized clinical trial. Intramuscular, multivalent, modified-live viral vaccination at 2 or 5 wk of age or both did not affect the incidence of respiratory disease and mortality, and growth before 3 mo of age on commercial dairy farms.

Effect of abomasal emptying rate on the apparent efficiency of colostral immunoglobulin G absorption in neonatal Holstein-Friesian calves. By Mokhber-Dezfooli et al., page 6740. Inadequate absorption of colostral immunoglobulin G ( $\operatorname{IgG}$ ) in calves is an important source of economic loss to the dairy industry. We investigated whether an increase in the rate of abomasal emptying in colostrum-fed calves was associated with an increase in the apparent efficiency of absorption (AEA) of colostral IgG. Twenty-four neonatal Holstein-Friesian calves were fed $3 \mathrm{~L}$ of pooled cow colostrum containing acetaminophen $(50 \mathrm{mg} / \mathrm{kg}$ of body weight) by oroesophageal intubation. Abomasal emptying rate was varied by administering erythromycin, ivermectin, gentamicin, or placebo. Jugular venous blood samples were obtained periodically after the start of feeding, and abomasal emptying rate was assessed by the time to maximal plasma acetaminophen concentration. Using data from all 24 calves, the AEA of colostral IgG was linearly and negatively associated with abomasal emptying rate. We conclude that abomasal emptying rate is an important determinant of the AEA of colostral IgG in calves.

Short communication: Survival, growth to weaning, and subsequent fertility of live-born dairy heifers after a difficult birth. By Barrier et al., page 6750 . The longer term effects of experiencing a difficult birth were investigated in dairy heifers. Live-born dystocial heifer calves had poorer survival both in the neonatal period and until first service. Their growth to weaning and subsequent fertility were not affected, either because performance of weaning survivors was not affected or because it was compensated for by farm management. The higher mortality of dystocial heifer calves beyond the neonatal period stresses the importance of preventing dystocia. This would improve farm efficiency and dairy cattle welfare.

Short communication: In vivo deposition of $\left[1-{ }^{13} \mathrm{C}\right]$ vaccenic acid and the product of its $\Delta^{9}$ desaturation, $\left[1-{ }^{13} \mathrm{C}\right]$ rumenic acid, in the body tissues of lactating goats fed oils. By Toral et al., page 6755. Tracer methodology has been used to measure the relative contribution of in vivo $\Delta^{9}$-desaturation to cis-9,trans-11 conjugated linoleic acid (CLA) found in milk. However, the relative contribution of body tissues to the endogenous synthesis of cis-9,trans-11 CLA in lactating ruminants is not known. This study provides the first measurements of in vivo deposition of vaccenic acid (trans-11 18:1) and the product of its 
$\Delta^{9}$-desaturation, cis-9,trans-11 CLA, in mammary secretory tissue, liver, and omental and perirenal adipose tissues in goats fed 2 dietary treatments and examines the potential link with the expression of genes encoding $\Delta^{9}$-desaturase (SCD1 and $\left.S C D 5\right)$.

Composite $\beta$ - $\kappa$-casein genotypes and their effect on composition and coagulation of milk from Estonian Holstein cows. By Vallas et al., page 6760. Different genetic variants of the major milk protein, casein, have been found to influence milk coagulation and composition traits. This therefore enables the genetic improvement of milk to increase the efficiency of the dairy industry. The effect of composite $\beta$ - $\kappa$-casein genotypes on milk coagulation and composition was studied using a random regression animal model. The $\beta$ - $\kappa$-casein genotypes affecting milk coagulation traits were identified and their effect on genetic variability was reported.

Relationship between body condition score and health traits in first-lactation Canadian Holsteins. By Loker et al., page 6770. In the last decade, interest has increased in improving health and fertility traits in the Canadian dairy cattle population. Indicator traits such as body condition score may be more useful than selecting directly for functional traits because it has a higher heritability and is easier to measure. This study found that increased body condition score was genetically associated with reduced mastitis and metabolic disease (displaced abomasum, milk fever, and ketosis) throughout first lactation in Canadian Holsteins.

Genetic and nongenetic variation in plasma and milk $\beta$-hydroxybutyrate and milk acetone concentrations of early lactation dairy cows. By van der Drift et al., page 6781. This study investigated differences in susceptibility of dairy cows to hyperketonemia (ketosis) by estimating genetic variation and heritability for concentrations of plasma $\beta$-hydroxybutyrate (BHBA), milk BHBA, and milk acetone at test days in early lactation. Heritability estimates of plasma BHBA, milk BHBA, and milk acetone were 0.17, 0.16, and 0.10 , respectively. Correction for expected milk production level influenced heritability estimates to a minor extent, suggesting that hyperketonemia susceptibility is not strongly associated with milk production potential. Genetic variation in routinely analyzed milk BHBA and milk acetone exists and can contribute to genetic improvement of susceptibility to hyperketonemia.

Short communication: Genetic parameters for feed intake, production and extent of negative energy balance in Nordic Red dairy cattle. By Liinamo et al., page 6788. Dairy cows often cannot obtain enough energy from their feed and need to utilize their own body energy reserves for production. This negative energy balance can predispose cows to various health problems. Breeding for improved feed intake could alleviate the negative energy balance, but it could also lead to correlated increases in body weight. Direct selection for energy balance in early lactation might be a better option, but would still require feed intake data for selection candidates.

Short communication: CSN1S1-CSN3 $\left(\alpha_{\mathrm{S} 1-\kappa-}\right.$ casein) composite genotypes affect detailed milk protein composition of Mediterranean water buffalo. By Bonfatti et al., page 6801. In bovine milk, protein composition is known to affect coagulation properties, and casein genes are responsible for considerable variation in protein composition. The effects of casein genes on the protein composition of buffalo milk are unknown. This study provides evidence that protein composition of Mediterranean water buffalo milk; namely, the percentages of $\alpha_{\mathrm{S}_{1}-} \mathrm{CN}, \alpha_{\mathrm{S}_{2}} \mathrm{CN}$, and $\kappa-\mathrm{CN}$ in total casein and the proportion of glycosylated $\kappa-\mathrm{CN}$ in total $\kappa-\mathrm{CN}$, is influenced by CSN1S1-CSN3 composite genotypes, which might be used in geneassisted selection to alter protein composition.

Comparison between mechanical and nearinfrared methods for assessing coagulation properties of bovine milk. By Cipolat-Gotet et al., page 6806. Milk coagulation properties are important in cheese making. Traditional parameters obtained from mechanical devices such as the Formagraph and the computerized renneting meter are rennet coagulation time, curd-firming time, and curd firmness. Recently, new optical devices have been developed for assessing these properties, as an alternative to mechanical devices. In the present study, we compared the results from mechanical and optical instruments under identical conditions. We found differences between instruments, especially when late-coagulating milk samples were detected. Findings suggest that the optical devices process raw data in a manner that differs from that dictated by the algorithms of the mechanical instruments.

Producer survey of bird-livestock interactions in commercial dairies. By Shwiff et al., page 6820. Wild birds are known to feed on cattle rations within dairies. Researchers have speculated that their presence may cause economic damage and veterinary health risks. Currently, few data are available to help dairies identify whether problems exist or what to do when bird damage is known to occur. To better understand bird-livestock interactions in dairies, we surveyed operations in Pennsylvania, New York, and Wisconsin. This information was used to identify bird species, areas of economic damage, and whether veterinary health risks are created through bird use of dairies. 